\title{
Method for Image Restoration using Wavelet based Image Fusion
}

\author{
Arun Kumar Patel, Nitin Muchhal \\ Astt Prof, Deptt of ECE \\ Sagar Institute of Science \& Technology \\ Bhopal, (MP) India- 462036
}

\author{
R.N. Yadav \\ Prof, Deptt of ECE \\ M.A.N.I.T, \\ Bhopal, India 462003
}

\begin{abstract}
With the increase demand of better image quality lot of image processing algorithms are designed. In this paper we described a method to remove the motion blur present in the image taken form any cameras. Motion blur is the result of the relative motion between the camera and the scene during image exposure time, this includes both camera and scene objects motion.. The aim of image restoration is to reconstruct or estimate an uncorrupted image by using the degraded version of the same image. [2]

The most closely related research can be found in Blind deconvolution, and Wiener filter methods, They adopts regularized iteration to restore the degraded image. This work proposes the implementation of Wiener filter with Image fusion to reduce the computational complexity with better acceptable restoration results of image restoration method.. The performance of the every stage is tabulated for the parameters like SNR and RMSE of the restored images.
\end{abstract}

Key Words: Image restoration, Point Spread Function, Motion blur, Wirner Filter, WT Image Fusion

\section{INTRODUCTION}

Images acquired through any modern sensors consist of variety of noises (such as thermal noise, amplifier noise, photon noise, quantization noise and cross talk), resulting from stochastic variations and deterministic distortions or shading. In order to extract intrinsic image data from raw measured image data, smoothing algorithms should be applied initially to reduce noises and to preprocess images for further analysis and processing. For slowly varying noise, a linear filter can be selected for noise smoothing. For rapidly varying noise, a nonlinear filter can be applied.

Motion blur[9] occurs in many image formation systems due to limited performance of both optical and electronic systems [8]. The shutter speed, for example, is the main factor deciding the amount of motion blur. As blurring can significantly degrade the visual quality of images, with a lowcost camera Photographers and camera manufactures are frequently searching for methods to limit the phenomenon.

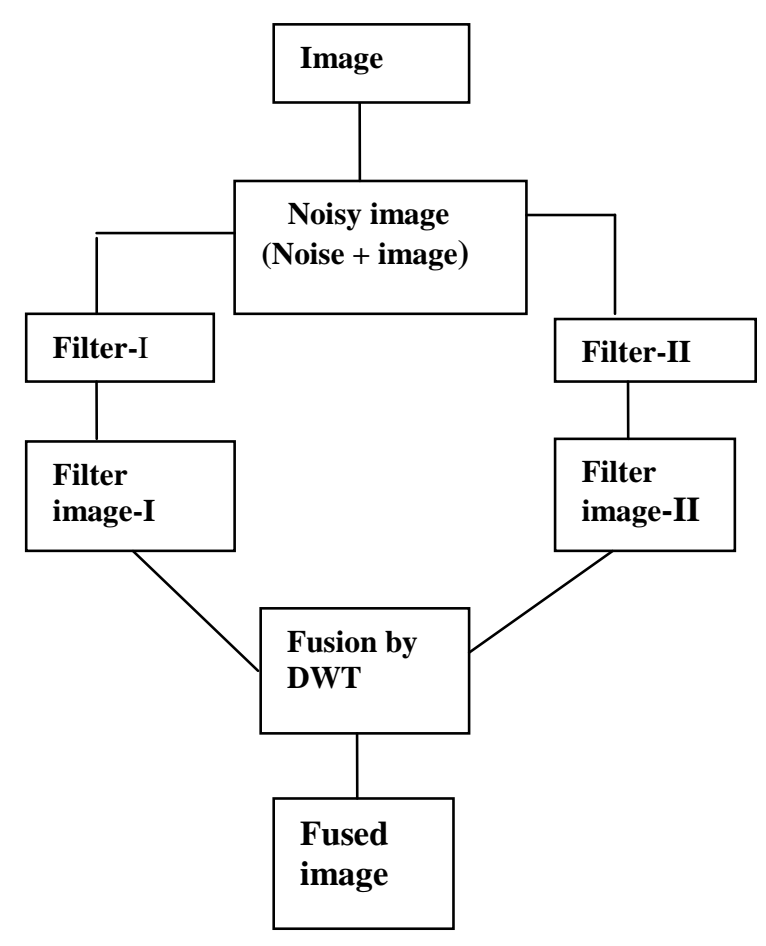

Fig.1 Block Diagram

One solution that reduces the degree of blur is to capture images using shorter exposure intervals. This, however, increases the amount of noise in the image, especially in dark scenes. An alternative approach is to try to remove the blur off-line. Various image restoration methods have been studied for removing motion blur such as iterative method. However, their computational complexity such as regularized iteration is so high that they can hardly be implemented in real-time [1] .If shutter speed is relative to the object velocity, the obtained image is degraded by motion blur. Blur is usually modeled as a linear convolution of an image with a blurring kernel, also known as the point spread function (or PSF) [3] . In Remotely sensed images the relative motion is slow it results in fatal failure of detecting important information. Because of this reason, approach is to try to remove the blur off-line.

The purpose of image restoration is to reconstruct or estimate an uncorrupted image by using the degraded version of the same image. One of the most common degradation functions is linear motion blur with additive noise eq.1.1 shows the relationship between the observed image $\mathrm{g}(\mathrm{x}, \mathrm{y})$ and its uncorrupted version $\mathrm{f}(\mathrm{x}, \mathrm{y})[5]$ 


$$
g(x, y)=f(x, y) * h(x, y)+\eta(x, y) .
$$

In this equation, $\mathrm{h}(\mathrm{x}, \mathrm{y})$ is the blurring function (or point spread function (PSF)), that is, convolved with the original image $f(x, y)$ and $n(x, y)$ is the additive noise function. According to eq.1.1 in order to determine the uncorrupted image, we need to find the blurring function $\mathrm{h}(\mathrm{x}, \mathrm{y})$ (i.e., blur identification), which is an ill-posed problem The degraded image in low-frequency region however, will be similar to the restored image. The first stage compared the two methods of wiener filter and blind de-convolution. The blind deconvolution is an iterative method and requires more computation. The wiener filter is a minimum mean square error method, but results have some shadowing effects. So in first stage some better results are formed but the blur is not completely removed.

Extracting more information from multi source images is an attractive thing in remotely sensed image processing, which is recently called image fusion. There are many image fusion methods so far, such as WS. PCA. WT. GLP etc,.here we adopt wavelet fusion method So the resultant image is not accurate. The development packages investigated for the development of the research application was MATLAB[9], [10,] which is commercially available and run on Microsoft Windows.

\section{IMAGE RESTORATION}

The aim and objective of image restoration is to reconstruct or estimate an uncorrupted image by using the degraded version of the same image[5],. Thus restoration techniques are oriented toward modeling the degradation and applying the inverse process in order to recover the original image.

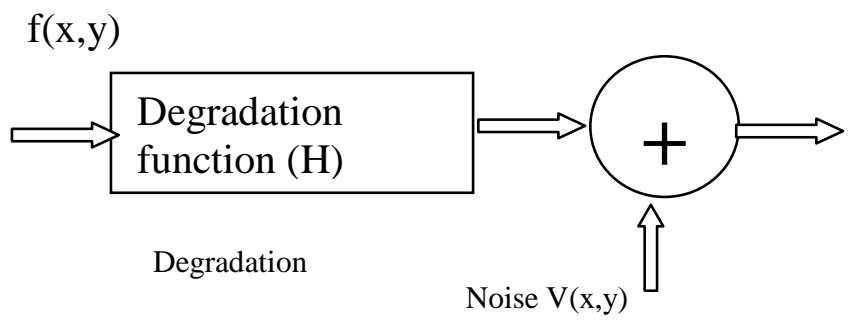

Fig 2. Image Degradation Model

This approach usually involves formulating a criterion of goodness that will yield an optimal estimate of the desired result. The image gets blurred due to the degradation. Blur is of many types but for our work we have considered the motion blur only.

\subsection{Image Degradation Model}

The image degradation model is the mathematical representation of the way in which the image gets blurred [3]. There is different image degradation models are proposed for different purpose. Image degradation model for motion blur is formulated as the eq. (1)

Where $G(x, y)$ represents the observed image.(where $\mathrm{x}$ and $\mathrm{y}$ are the coordinates of the image pixel). We consider $\mathrm{f}(\mathrm{x}, \mathrm{y})$ as original image degraded by the point spread function $\mathrm{H}(\mathrm{x}, \mathrm{y})$, with noise $\mathrm{V}(\mathrm{x}, \mathrm{y})$.If $\mathrm{H}$ is a linear spatially invariant process, then the degraded image is given by eq.(1). The.convolution in spatial domain and multiplication in the frequency domain constitute a Fourier transform pair so the eq (2) may be modified as

$G(u, v)=F(u, v) * H(u, v)+N(u, v)$

The degradation function $\mathrm{H}(\mathrm{u}, \mathrm{v})$ is some times are also called the optical transfer function (OTF), a term derived from the Fourier analysis of the optical system. In the spatial domain, $\mathrm{h}(\mathrm{x}, \mathrm{y})$ is referred as the point spread function (PSF). The OTF and PSF are the Fourier transform pairs. Generally the $\mathrm{V}$ is modeled as zero mean white Gaussian additive noise. But here we have modeled noise as sum of the multiplicative noise and additive Gaussian noise as

$\mathrm{V}(\mathrm{x}, \mathrm{y})=\mathrm{f}(\mathrm{x}, \mathrm{y})^{*} \eta 1(\mathrm{x}, \mathrm{y})+\eta 2(\mathrm{x}, \mathrm{y})$

Where $\eta 1(x, y)$ is the multiplicative noise and $\eta 2(x, y)$ is the additive noise.

$\mathrm{g}(\mathrm{x}, \mathrm{y})$

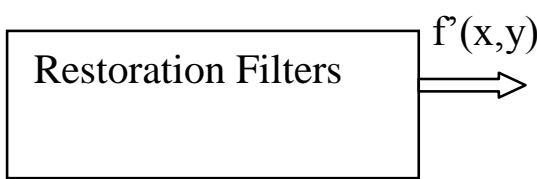

\subsection{Blind Deconvolution}

De-convolution is a signal-processing operation that, ideally, unravels the effects of convolution performed by a linear time-invariant system operating on an input signal. In deconvolution, the output signal and the system are both known and the requirement is to reconstruct what the input signal must have been. In blind deconvolution, only the output signal is known (both the system and the input signal are unknown), and the requirement is to find the input signal and the system itself. But it needs higher Computation So it is lengthy process for real time systems.

\subsection{Wiener Filter}

The wiener filter is termed as minimum mean square error filter. Neglecting the non-diagonal terms of the covariance matrix of $\mathrm{v}(\mathrm{x}, \mathrm{y})$, and using the fact that $\sigma 22<$ $\sigma 12$ we obtain the Wiener filter estimate of the blur PSF

$$
D(\omega)=\frac{\alpha N^{*}(\omega) G(\omega)}{\alpha^{2} \mathrm{~N}(\omega)+\sigma_{1}}
$$

Where the capital letters stand for the Fourier transforms of the corresponding noise and original signal, and the value of $\alpha$ in the first iteration, can be obtained as the ratio between the means of the two images: and is calculated using the estimator

$$
\alpha=\frac{\text { Mean of } \mathrm{v}(\mathrm{x}, \mathrm{y})}{\text { Mean of } \mathrm{g}(\mathrm{x}, \mathrm{y})}
$$

Then parameter $\alpha$ is estimated at each iteration by equating equation (4) with zero this is An initial estimate of the blur PSF is there by obtained from the inverse Fourier transform of equation (4) 


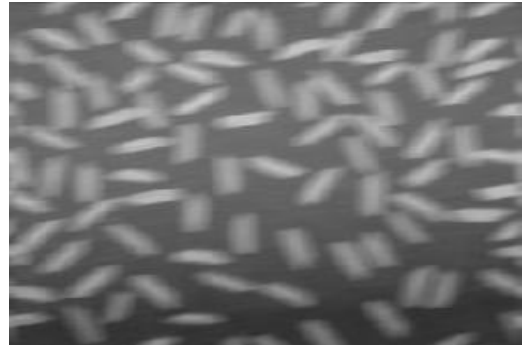

a)Blurred Noisy Rice Image

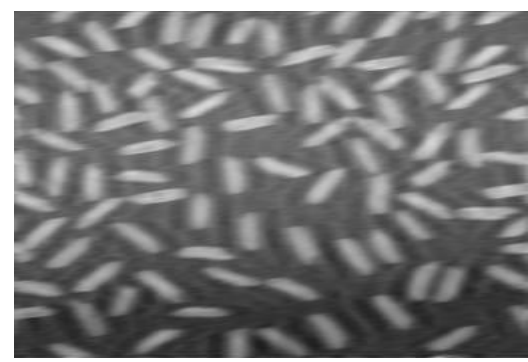

b) Restored Image by Wiener Filter with ratio $\mathbf{R}$ Fig. 3 Filtering Result

\section{POINT SPREAD FUNCTION (PSF)}

The General form of motion blur function is given as follows

$$
H(x, y)=\left\{\begin{array}{cc}
\frac{1}{L} & \text { if } \sqrt{x^{2}+y^{2}} \frac{L}{2}, \frac{X}{Y}=-\tan (?) \\
0 & \text { elsewhere }
\end{array}\right.
$$

As seen that motion blur function depends on two parameter Motion length L \& Motion Direction Ǿ

\section{IMAGE FUSION}

Extracting more information from multi source images is an attractive thing in remotely sensed image processing, which is recently called data fusion[6]. There are many image fusion methods so far, such as WS. PCA. WT. GLP etc. Among these methods WT and GLP methods can preserve more image spectral characters than others. So here we adopt wavelet method (as it is proposed to improve the geometric resolution of the images).

Image fusion is the process of combining two or more source images into composite images with extended information content
Image fusion technique [7] is a powerful tool for extracting high quality information from large number of remotely sensed images and limiting redundancy among these images. We use WT Fusion.

\subsection{Wavelet Based Image Fusion}

Wavelet transform [5],[10], [12] is first performed on source images. Then a fusion decision map is generated based on a set of fusion rules. Then fused wavelet coefficient map can be constructed from the wavelet coefficients of the source images according to the fusion decision map. Finally the fused image is obtained by performing the inverse wavelet transform. We use pixel level maxima algorithm simplicity.

\subsection{Pixel Level Maximum (PLM)}

All the four sub bands of the fused image [2], [8] $\mathrm{F}$ is simply formed by taking the wavelet coefficients from source images which is having the maximum value. $\mathrm{Fj}, \mathrm{k}=\max (\mathrm{Aj}, \mathrm{k} B \mathrm{Bj}, \mathrm{k}) \& \mathrm{FJ}=\max (\mathrm{Aj}, \mathrm{Bj})$.

\section{RESULT AND CONCLUSION}

In paper section, we present some experimental results of our work on wavelet biased, Proposed method and, together with, blind- de-convolution and wiener filter methods and analysis is done on the basis of the mean square error and signal to noise ratio

In the first stage result, We can see that the results based on the suggested wiener filter provides the better results than iterative blind de convolution method .The results are compared on the basis of the Signal to Noise ratio (SNR) \& Root Mean Square Error (RMSE), for Different image size ( $64 \times 64,128 \times 128,128 \times 256,256 \times 256,512 \times 512)$. It is found that restoration with fusion is faster and gives better results for most cases.

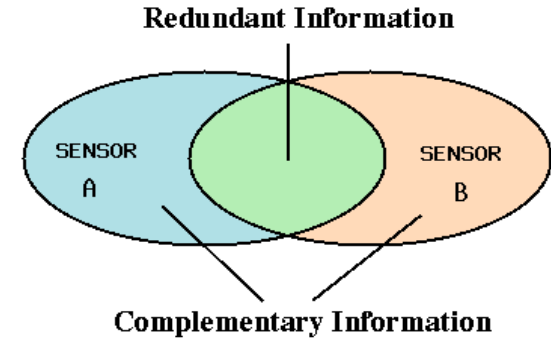

Fig 4. Image fusion 


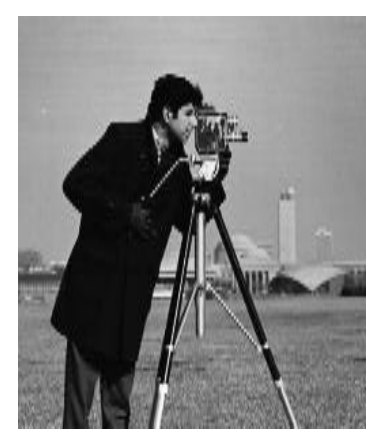

a) Original image

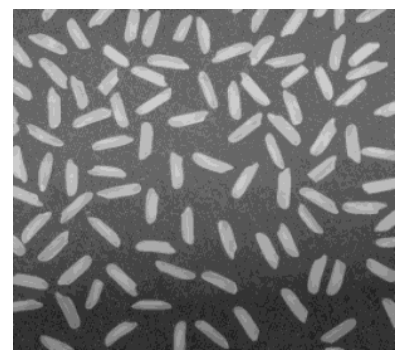

a) Original image

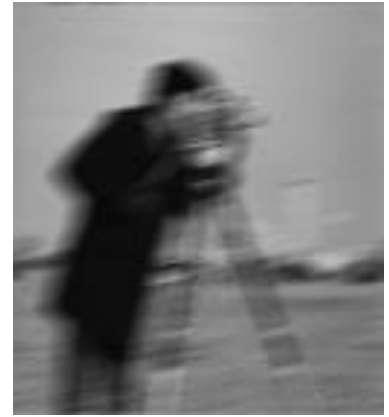

b) Blurred noisy image

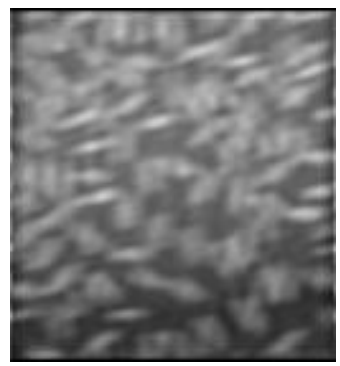

b) Blurred noisy image

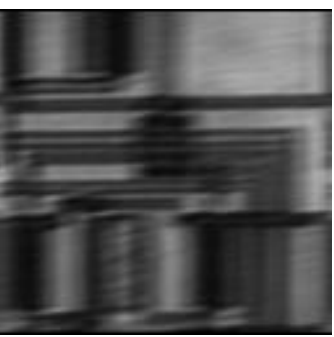

b) Blurred noisy image

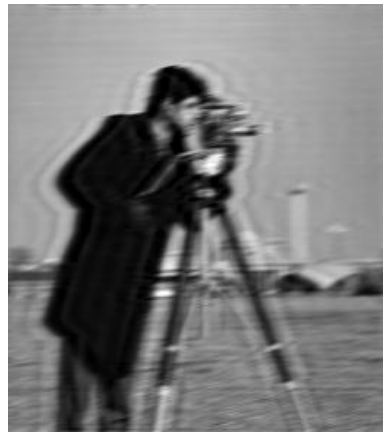

c) Restored image

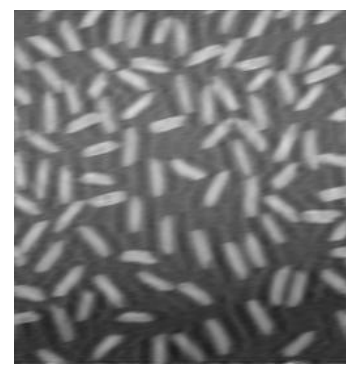

c) Restored image

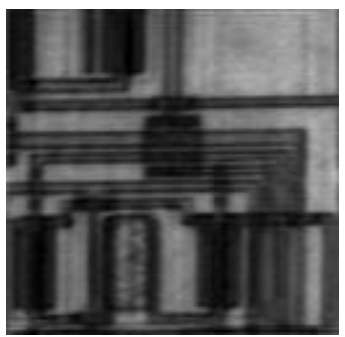

c) Restored image

(a) Original circuit image

Figure 5: Comparison of original and restored image for various images

TABLE-.1 Comparison of RMSE between Blind-Deconvolution and Wiener Filter (For Circuit Image)

\begin{tabular}{|c|c|c|c|c|c|}
\hline S.No. & Size Of Image & Blured Noisy Image & \multicolumn{2}{|c|}{$\begin{array}{c}\text { Blind } \\
\text { De-Convolution }\end{array}$} & Wiener Filter \\
\hline & & & $\mathrm{N}=5$ & $\mathrm{~N}=10$ & WITH RATIO R \\
\hline 1 & $64 \mathrm{X} 64$ & 28.199 & 31.645 & 33.793 & 11.245 \\
\hline 2 & $128 \mathrm{X} 128$ & 19.287 & 21.512 & 23.246 & 10.509 \\
\hline
\end{tabular}




\begin{tabular}{|c|c|c|c|c|c|}
\hline 3 & $128 \times 256$ & 13.928 & 16.849 & 17.972 & 8.4339 \\
\hline 4 & $256 \times 256$ & 12.961 & 14.523 & 15.241 & 9.1132 \\
\hline 5 & $512 \times 512$ & 8.4039 & 9.5547 & 9.7914 & 7.3573 \\
\hline
\end{tabular}

TABLE-2 Comparison of SNR between Blind-deconvolution and Wiener filter (for Circuit Image )

\begin{tabular}{|c|c|c|c|c|c|}
\hline S.No. & Size Of Image & Blured Noisy Image & \multicolumn{2}{|c|}{$\begin{array}{c}\text { Blind } \\
\text { De-Convolution }\end{array}$} & Wiener Filter \\
\hline & & & $\mathrm{N}=5$ & $\mathrm{~N}=10$ & $\begin{array}{c}\text { WITH RATIO } \\
\mathrm{R}\end{array}$ \\
\hline 1 & $64 \mathrm{X} 64$ & 3.1775 & 2.8315 & 2.6515 & 7.9681 \\
\hline 2 & $128 \mathrm{X} 128$ & 4.6409 & 4.161 & 3.8507 & 8.5179 \\
\hline 3 & $128 \times 256$ & 6.4181 & 5.3057 & 4.9747 & 10.6 \\
\hline 4 & $256 \times 256$ & 6.886 & 6.1477 & 5.8577 & 9.7967 \\
\hline 5 & $512 \times 512$ & 10.648 & 9.3654 & 9.1389 & 12.163 \\
\hline
\end{tabular}

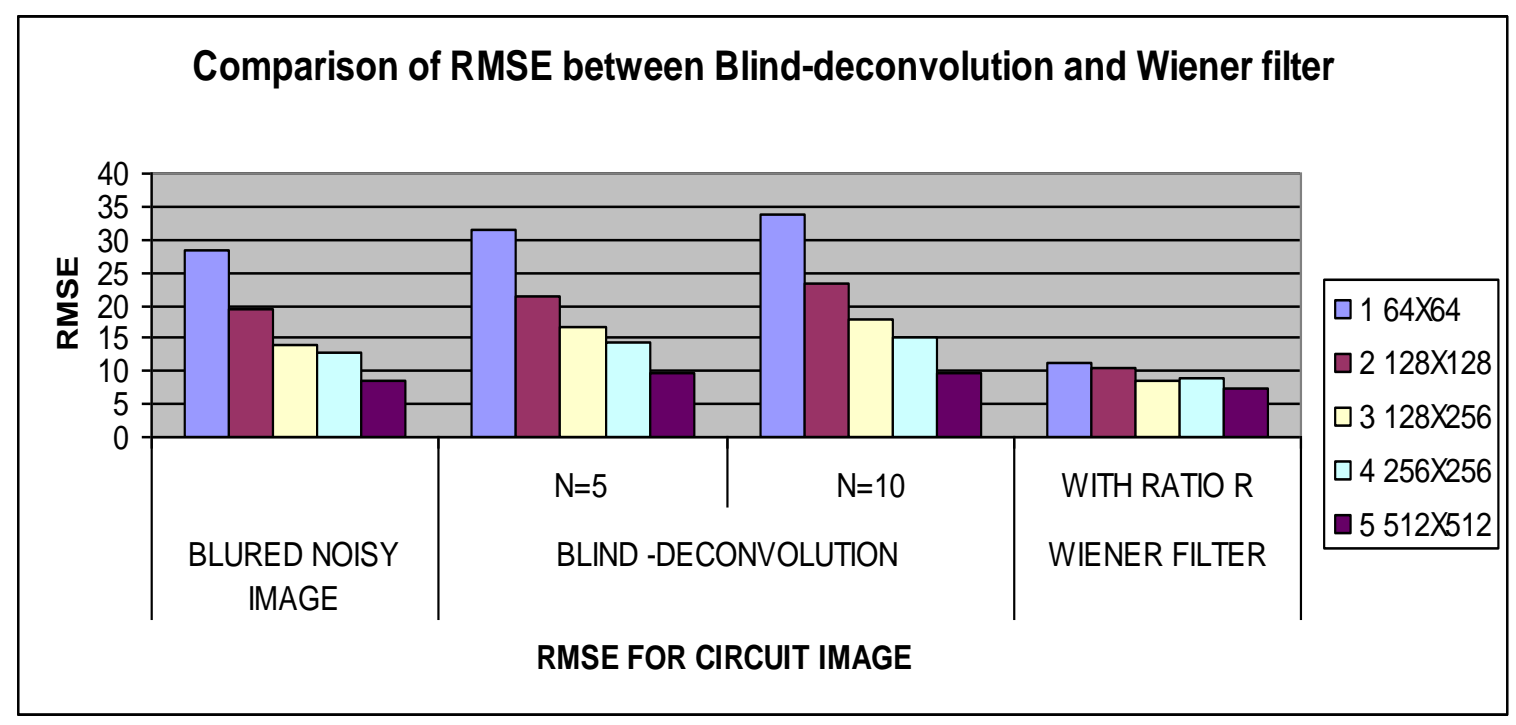




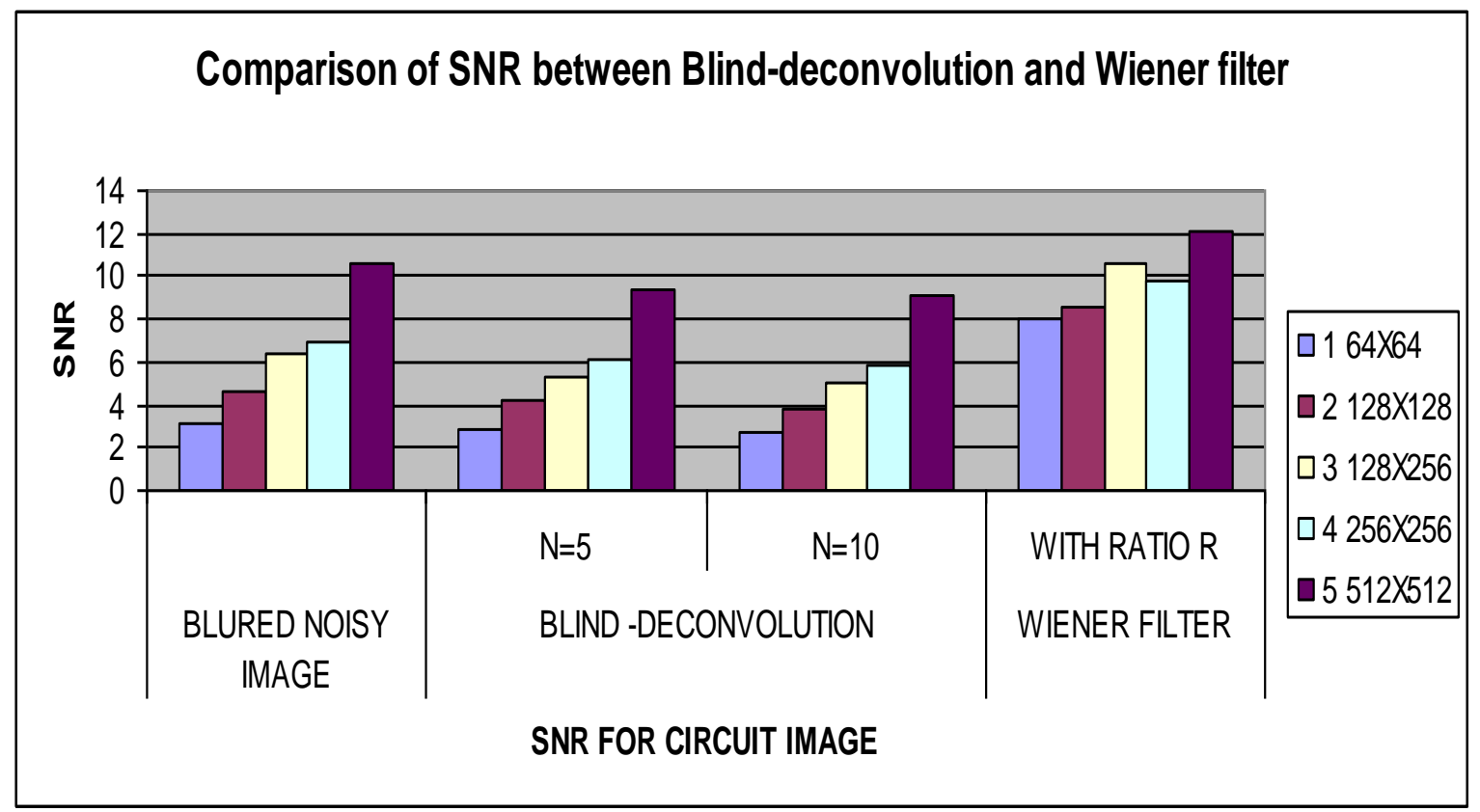

\section{CONCLUSION}

This work implemented and compared the performance with Wiener filter and Blind de convolution as shown with concluding table. We find from results that Weiner filter with Image fusion reduce the computational complexity with better acceptable restoration results of image restoration tabulated for the parameters like SNR and RMSE of the restored images.

\section{REFERENCES}

[1] W. Dong, L. Zhang, G. Shi and X. Wu, "Image deblurring and supper-resolution by adaptive sparse domain selection and adaptive regularization," in IEEE Trans. on Image Processing, 2011., vol. 20, no. 8, pp. 2378-2386, 2011.

[2] Deepa Li A. Godse Dattatraya S. Bormane" Wavelet Based Image Fusion Using Pixel Based Maximum Selection Rule" International Journal of Engineering Science and Technology (IJEST) Vol. 3 No. 7 July 2011

[3] S. K. Sathpathy, S. Panda, K. K. Nagwanshiand C. Ardil, "Image Restoration in Non-Linear Filtering Domain using MDB approach", International Journal of SignalProcessing, 2010.

[4] Weisheng Dong, Lei Zhang, Guangming Shi, Xiaolin Wu, "Nonlocal back-projection for adaptive image enlargement," ICIP 2009.

[5] M.S.Alani, Digital Image Processing using Matlab, University Bookshop, Sharqa, URA, 2008.
[6] M.A.Joshi, Digital Image Processing - An Algorithmic Approach, Professors and Head, Department of Telecommunications, College of Engineering, Pune,Prentice Hall of India Private Limited, New Delhi, 2007.

[7] Youshen Xia, and Mohamed S. Kamel," Novel Cooperative Neural Fusion Algorithms for Image Restoration and Image Fusion"Feb, 2007.

[8] Zing, J.; Sayedelahl, A.; Gilmore, T.; Chouikha, M," Review of Image Fusion Algorithms for Unconstrained Outdoor Scenes" Signal Processing, The 8th International Conference on Volume 2, Issue 2006

[9] Marius Tico, Sakari Alenius, and Markku Vehvi- lainen, "Method of motion estimation for image sta- bilization," in IEEE International Conference on Acoustics, Speech, and Signal Processing (ICASSP), Toulouse, France, 2006.

[10] Rafael C. Gonzalez, Richard E. Woods, Steven L. Eddins, "Digital Image Processing Using MATLAB." Pearson, Third Edition 2005.

[11] Ajay V. Deshmukh, Vivek Shivhare, "Periodicity Analysis of FMRI data in the Wavelet Domain", National Communications Conference, 2005 at the Indian Institute of Technology, (IIT) Kharagpur 2005.

[12] Vikramsingh K. Roday, Vikram M. Gadre, Arun N. Chandorkar and Nabar Vidyesh, "Efficient VLSI Implementation of 2-D Discrete Wavelet Transform for JPEG2000 Standard", Asia and South Pacific International Conference on Embedded SoCs, IISc Bangalore, July 2005. 